\title{
Magnetc Torque in Superoxide Ion is the Main Driving Force of Dioxygen Activation in Aerobic Life
}

\author{
Boris Minaev* \\ Department of Chemistry and Nanomaterials Science, The Bohdan Khmelnitsky National University, Ukraine \\ *Corresponding author: Boris Minaev, Department of Chemistry and Nanomaterials Science, The Bohdan Khmelnitsky \\ National University, Ukraine
}

\section{ARTICLE INFO}

Received: August 14, 2021

Published: September 03, 2021

Citation: Boris Minaev. Magnetc Torque in Superoxide Ion is the Main Driving Force of Dioxygen Activation in Aerobic Life. Biomed J Sci \& Tech Res 38(4)-2021. BJSTR. MS.ID.006171.

\begin{abstract}
Dioxygen $\left(\mathrm{O}_{2}\right.$ molecule $)$ is a biradical with two unpaired electron spins, while most of organic substances have all electron spins paired; therefore reactions catalysed by oxidases and oxygenases have to involve intersystem crossing (ISC), that is a total spin change in the state multiplicity during bio oxidation. These reactions are strictly forbidden in the ordinary non-relativistic quantum chemistry. In order to overcome this severe quantum prohibition and sovereign limitation of dioxygen-induced metabolism, oxygenation enzymes require usually a redox cofactor or paramagnetic metal cofactor for effective catalysis. The spin-containing metal ions have no formal spin prohibition for reactions with dioxygen. But many oxidative enzymes include the metal-free flavin or pterin cofactors; their catalytic activity in respect to $\mathrm{O}_{2}$ still remains mysterious in modern biochemistry and medicine. Last decades have shown, that some oxygenases can catalyse dioxygen incorporation into organic substrates even in the absence of any enzymatic cofactor. The intriguing mechanism followed by those cofactor-free enzymes which enable to provide ISC and overcome spin prohibition has been unraveled recently on the ground of common idea about specific magnetic properties of dioxygen open shell. Similar spin-orbit coupling effects are typical for $\mathrm{O}_{2}$ molecule and for some reactive oxygen species (ROS). These spin-orbit torques are vitally decisive forces for all aerobic life.

Abbreviations: ISC: Intersystem Crossing; ROS: Reactive Oxygen Species; HOMO: Highest Occupied Molecular Orbital; NIR: Near Infrared; NHE :Normal Hydrogen Electrode; RP: Radical Pair; T-S: Triplet Singlet; S: Spin; BYA: Billions Years Ago; GOE: Great Oxygen Event; OP: Oxidative Phosphorilation; ATP: Adenosine Triphosphate; SOD: Superoxide Dismutase; FAD: Flavin Adenine Dinucleotide; FMN: Flavin Mononucleotide; GO: Glucose Oxidase; DFT: Density Functional Theory; RPT: Radical Pair Theory; MO : Molecular Orbitals
\end{abstract}

\section{Introduction}

Oxygen produced by photosynthesis is everywhere - in air, in water and inside all aerobic creatures including ourselves. It plays such a great role in all biosphere that it is called "a wonderful elixir of life from the green leafs under the Sun" [1]. The main factor, which determines all wonderful properties of oxygen being the origin of aerobic life on the Earth can be expressed in one word and this word is spin. Electronic spin of the oxygen molecule explains the origin of all paradoxes in physics and chemistry of this simple diatomic species including its importance for living matter [119]. At the same time the well-known chemical concepts which generalize the role of electron spin in chemical reactions [14] are not fully applied for bio-oxidation processes [1]. Respiration and combustion are known to be equivalent in the balance of their exothermicity and the yield of reaction products; all carbohydrates and hydro-carbons are oxidized by molecular oxygen to $\mathrm{H}_{2} \mathrm{O}$ and $\mathrm{CO}_{2}$. Ignition of organic fuel in the open air provides occurrence of 
the first radicals, which can initiate a radical-chain process thereby removing the spin-forbidden character of $\mathrm{O}_{2}$ reaction with organic substances. Such type of dioxygen activation is impossible in-side the living cell since the radicals would burn the cell.

Aerobic organisms use special enzymes, which involve magnetic perturbations affording to induce ISC and brake the severe spin selection rule for two unpaired electrons of dioxygen during bio-oxidation of organic matter; that is to overcome spin selection and sever quantum prohibition for triplet $\mathrm{O}_{2}$ biradical to react with diamagnetic organic substances. Magnetic spin catalysis of concerted reactions of molecular oxygen in the enzyme active center does not correspond to the classical chemical concepts and common organic reaction rules. One has to stress that only magnetic forces are able to induce spin flip, which separates the unpaired $\mathrm{O}_{2}$ electrons from the diamagnetic products of bio-oxidation $\left(\mathrm{CO}_{2}, \mathrm{~N}_{2}\right.$ and water molecules with all spins paired). Internal magnetic forces in molecules are much weaker than electric forces which govern all main chemical interactions; bond strength, heat of formation, dissociation and activation energy accompanied by the spin flip can be considered only with relativistic quantum chemistry methods. These quantum processes inside living matter do not obey the common chemistry rules and are determined by internal magnetic interactions being specific to the electronic open shell of dioxygen. They are important for all aerobic evolution, photo-synthesis, appearance of reactive oxygen species and superoxide dismutase, oxi-dative stress and signalling functions in plants.

The problem of molecular oxygen activation is a longstanding task in bio-chemistry [1-6]. In contrast to the majority of stable organic substances, M in Eq. (1), which usually are dia magnetics, since their electron spins are paired and the total spin quantum number $(\mathrm{S})$ is zero, the molecular oxygen (dioxygen) is a paramagnetic gas. That is, the $\mathrm{O}_{2}$ molecule has a ground triplet state $\mathrm{X}^{3} \Sigma_{\mathrm{g}}$ with a nonzero electron spin $(\mathrm{S}=1)$ produced by two nonpaired electrons at the degenerate highest occupied molecular orbital (HOMO) $\pi \mathrm{g}$ [1]. The presence of spin in the ground state of $\mathrm{O}_{2}$ leads to a strict quantum prohibition on oxidation of organic substrates by dioxygen, since the reaction products $\left(\mathrm{P}=\mathrm{CO}_{2}, \mathrm{H}_{2} \mathrm{O}\right.$, $\mathrm{N}_{2}$ ) are also diamagnetic species.

$$
O_{2}(\uparrow \uparrow)+M(\uparrow \downarrow)=P(\uparrow \downarrow)(\uparrow \downarrow)
$$

The $\mathrm{M}$ molecule has a singlet ground state $(\mathrm{S}=0)$ and the total spin of reactants is triplet $(S=1)$, while the product has a singlet state again. Thus, combustion of organic matter is strictly spinforbidden if no special initiation by additional radical $R$, which is responsible for ignition.

$$
O_{2}(\uparrow \uparrow)+M(\downarrow \uparrow)+R \bullet(\downarrow)=P(\uparrow \downarrow)(\uparrow \downarrow)+O O R \bullet(\uparrow)
$$

The total spin $(\mathrm{S}=1 / 2)$ and its projection on $\mathrm{z}$-axis $(\mathrm{Ms}=+1 / 2)$ are the same in the left and right part of Eq. (2). Thus, the radical- initiated combustion proceeds as radical-chain reaction until the whole fuel $\mathrm{M}$ would be exhausted or the chain-transfer radical would be saturated and removed by a scavenger [1]. In the absence of radicals the direct oxidation by dioxygen is spin-forbidden, Eq. (1); this is the reason why our world had not been burnt when photosynthesis starts to saturate the early Earth's atmosphere and primordial oceans. The newborn radicals OOR• can continue the chain reaction with dioxygen without spin prohibition. Reaction from Eq. (2) can be repeated with any peroxide or other available radical $\mathrm{R} \bullet[14]$. Usually, this is a branched chain reaction which provides exothermic effect in the form of flame. In the flame there are many various radicals and random collisions are possible in the plasma volume; the newborn radical, Eq. (2), escapes into the volume and its spin orientation is arbitrary for new collisions. Thus, it loses spin memory, which was created during radical appearance, Eq. (2). In a new subsequent collision with new $\mathrm{O}_{2}$ molecule a random spin orientation can be realized, since the former spin memory is lost. But it is not the case for biooxidation inside the cellular enzyme where all possible radicals, if any are born, cannot leave the active center; they keep their spin memory obtained during the birth moment. The "fiery" mechanism, Eq. (2), in the cell is impossible also because the energy supply cannot be proper regulated in radical-chain reaction.

At the same time, the spin selection determined by the triplet ground state of dioxygen leads also to forbidden optical transitions in the visible and near IR spectrum of the $O_{2}$ molecule [9-16]. By that reason our atmosphere is transparent to visible sun light, though the energies of the singlet excited states of dioxygen just correspond to the red and near infrared (NIR) range of electromagnetic waves. The singlet $O_{2}{ }^{1} \Delta_{g}$ oxygen (known as one of the most reactive oxygen species in the oxidative stress) is monitored now in tissues by its NIR luminescence at $1.27 \mu \mathrm{m}$ [9-12]. Analysis of dioxygen wave-functions and transition moments in the electronic $\mathrm{O}_{2}$ spectrum sheds light on the role of internal magnetic forces, which make it possible to overcome spin restrictions in both enigmatic phenomena - in light emission and in biological oxidation by dioxygen $[1,12]$.

\section{Oxidizing Power of Dioxygen and its Slaggish Reactivity without Enzymes}

We used to take our respiration for granted and do not care much about spin of dioxygen; although, we have to care. The high reduction potential of dioxygen causes its great oxidizing power [3]. Using only thermodynamic backgrounds one can predict that $\mathrm{O}_{2}$ is one of the best oxidizing agent [7]. But the triplet spin in the ground state of dioxygen prevents spontaneous oxidation of organic matter and its full combustion into puf of smoke and soot. Respiration of aerobic creatures consumes almost $90 \%$ of all dioxygen produced by photosynthesis and utilized in the Earth's biosphere [6,19]. In aerobic organisms about $80 \%$ of the consumed dioxygen is 
utilized during conversion of food (glucose) to the viable ATP energy sources. This oxidative phosphorilation is catalyzed finally by cytochrome c oxidase in most aerobic organisms $[5,18]$. This terminal step of respiration represents the four-electron reduction of dioxygen in order to produce two water molecules, Eq. $3[5,6]$ :

$$
\mathrm{O}_{2}+4 e^{-}+4 \mathrm{H}^{+}=2 \mathrm{H}_{2} \mathrm{O}
$$

The overall resulting reduction potential of reaction (3) is equal $+0.815 \mathrm{~V}$ versus NHE (normal hydrogen electrode); this means that such reduction is highly favored in terms of thermodynamics $[5,13$ 20]. Much of the rest of the consumed dioxygen besides respiration, Eq. 3, is utilized during metabolism for biosynthesis of proteins and other useful molecular substrates. This biosynthesis is catalyzed by monooxygenases or dioxygenases. With these enzymes one or two oxygen atoms from dioxygen molecule are incorporated into the final products, respectively $[17,18]$. The overall reduction, Eq (3), usually proceeds through a series of one and two electron transfer steps [2-5]. These reactions are strongly favored by reduction potentials expecting the one-electron reduction of $\mathrm{O}_{2}$ to produce anion-radical superoxide $\mathrm{O}_{2}^{--}[5,19]$. The late process has a reduction potential equals $-0.33 \mathrm{~V}$ versus NHE; this relatively low reduction potential for one-electron transfer to dioxygen is an important factors which limits the kinetic reactivity of $\mathrm{O}_{2}$ [5] But this is not the most important factor! The point is that the high oxidizing power stored up in $\mathrm{O}_{2}$ molecule cannot be realized until the first one-electron jump and reduction to $\mathrm{O}_{2}{ }^{--}$will be produced. And the following reaction step in the enzyme active center has to proceed in a concerted manner with the same reducing agent $\mathrm{E}$, which is responsible for electron transfer to $\mathrm{O}_{2}$. This means that the

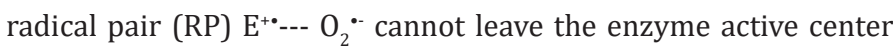
and the RP cannot dissociate before the spin-forbidden reaction in the triplet RP will occur [1-3]. The starting RP $\left(\mathrm{E}^{+\bullet---} \mathrm{O}_{2}^{*}\right)$ has the triplet ground state since the precursors $\left(\mathrm{E}+\mathrm{O}_{2}\right)$ have two non-paired spins in dioxygen moiety and the total spin $S=1$. Spin flip and triplet singlet (T-S) transition cannot be realized during electron transfer since the spin-conserving reduction step is much faster anywhere [13]. The necessary T-S transition can occur only at the geminate triplet radical pair step and this review is devoted to prove this statement.

The main paradox of life is that aerobic organisms cannot receive enough energy and survive without dioxygen, but at the same time $\mathrm{O}_{2}$ from the air is quite dangerous to their existence because of generation of reactive oxygen species (ROS) as byproducts of oxidative metabolism. This contradictory and inherent property of dioxygen relates to the triplet ground state of $\mathrm{O}_{2}$ molecule with the total spin ( $\mathrm{S}$ ) quantum number $\mathrm{S}=1$. This quantum number determines expectation value of the $S^{2}$ operator, where $\mathrm{S}=\mathrm{S}_{1}+\mathrm{S}_{2}$ is the total spin angular momentum for two electrons. Each electron has spin quantum number $S=1 / 2$ and two projection on the chosen z-axis $m_{s}= \pm 1 / 2$. The absolute value of $|S|$ or a length of the spin angular momentum vector of one electron is equal to $0.866 \hbar$. This value determines a strength of its magnetic field, which was measured and confirmed in numerous experiments [14]. For the triplet state $M s= \pm 1,0$ and we have three possible projection of the spin angular momentum on molecular axis in the $\mathrm{O}_{2}$ molecule. Not many biochemists had payed proper attention to this fundamental property of dioxygen, especially to its sequences for respiration and oxidative stress. Both mysterious phenomena are determined by magnetic perturbations in the $\pi \mathrm{g}$ open shell of dioxygen [1,14]. Thus, $\mathrm{O}_{2}$ is a stable free biradical and its reactions with organic molecules (which have the singlet ground state, $S=0$ ) are forbidden by severe spin selection rule. By this reason, dioxygen shows very sluggish chemical reactivity at ambient conditions (in the absence of radicals with unpaired electron spin) in spite of its strong thermodynamic potential for oxidation. This obstacle explains the second paradox of dioxygen reactivity; the high exothermicity of organic material oxygenation is in a great contradiction with a low reaction rate of their reactions with dioxygen in the absence of radicals [1]. According to the Polani Semenov rule the high exothermic effect of reaction correlates with its low activation barrier and the high reaction rate constant; the $\mathrm{O}_{2}$ reactivity demonstrates an opposite behavior [1]. Spin prohibition does not permit to realize the high oxidation power of dioxygen in terms of normal chemical kinetics [3]. That is why our world had not consumed into fire when green-blue algae started to saturate the Earth's atmosphere by photosynthetic dioxygen 2.4 billions years ago (BYA). That time is known as the Great Oxygen Event (GOE) or Oxygen Catastrophe, when anaerobic forms of life and archaea perished and were substituted by eukariots; thus, a new efficient type of aerobic evolution had started since then [8]. Before GOE the primordial atmosphere does not contain, or contains very little dioxygen. The notion of GOE is deeply imprinted now in our understanding of biosphere on the early Earth and of great role of this ecological catastrophe which ever shaked our planet. The impact of dioxygen spin state that time was much more important for living archaea than this spin is conceivable nowadays for the whole human civilization of man-kind.

A chronology of dioxygen accumulation on the Earth is rather complicated and includes about 900 million years from 3.3 to 2.4 BYA [20]. The increase of $\mathrm{O}_{2}$ concentration in primordial atmosphere, from $0.1 \%$ up to $15 \%$ during GOE, provided finally a great change in biochemical reactions responsible for energy supply from simple glycolysis in anaerobic bacteria to oxidative phosphorilation (OP) in eukariots as well as a new opportunity for cell proliferation and biological diversification. Anaerobic life based on glicolysis had been rather limited energetically (despite its efficient recycling of organic matter); only two ATP molecules are produced during glucose conversion into two molecules of 
pyruvate in the absence of $\mathrm{O}_{2}[5,6]$. Glycolysis still exists and takes place in the cytosol of modern eukariots in some emergency cases. But it was the only source of energy until the GOE time when $\mathrm{O}_{2}$ gas became widespread available on the Earth in air and oceans. The tricarboxylic acid (Krebs) cycle using dioxyen provides 32 ATP molecules, instead of two. Thus, much more free energy of adenosine triphosphate (ATP) became available to plants and animals and provided a breakthrough in evolution [5]. The advent of triplet dioxygen increased aerobic metabolism dramatically and made global impacts on all environment. What was the role of $\mathrm{O}_{2}$ electron spin in this Great Oxidation Event? Let us try to collect first the well-known facts.

One can consider mitochondria created and evolved after the GOE [5]. These mitochondria gave to aerobic cells much more energy exploiting oxidative phosphorilation with new and more complex morphology of inner membranes [5]. These are the places of the tricarboxylic acid cycle and the OP realization by which a large number of ATP molecules are produced from organic fuel. This is done through the electrochemical proton gradient, which is generated across the inner membranes by the high-energy electrons, which are passing along the electron transport chain [6]. But the role of mitochondria in living organisms extends far beyond oxidation of glucose via oxidative phosphorilation; it includes synthesis of haem, hormones, amino acids and many other molecules used for metabolism $[7,18]$. The recently discovered new role of mitochondria is their involvement in apoptosis and ion homeostasis through the signaling functions of ROS [8]. During the OP process mitochondria utilize dioxygen to generate ATP, but conjugation of this process with the electron transport chain can lead to $\mathrm{O}_{2}$ partial reduction and to superoxide anion $\left(\mathrm{O}_{2}{ }^{--}\right)$ generation. This is the most important reactive oxygen species being the ancestor of other ROS, which are partly reduced forms of dioxygen: the hydrogen peroxide $\left(\mathrm{H}_{2} \mathrm{O}_{2}\right)$, very reactive hydroxyl $\left(\mathrm{OH}^{\circ}\right)$, peroxyl radicals (ROO') and the singlet $\mathrm{O}_{2}{ }^{1} \Delta_{g}$ dioxygen [8]. Highly likely, that ROS appeared on the Earth during GOE together with the first photosynthetic dioxygen and ROS have been a permanent companion of aerobic evolution ever since [8].

In addition, one can take into account the highly-reducing milleu and high lev-el of reduced iron in the ancient oceans which provide efficient conversion of the first photosynthetic dioxygen into ROS [8]; probably, by this reason the former anaerobic life had perished. Dioxygen reduction in that milleu produced superoxide anion, which triggered other processes of ROS proliferation. The $\mathrm{O}_{2}$-- anion dismutates to form $\mathrm{H}_{2} \mathrm{O}_{2}$, which interacts with the soluble $\mathrm{Fe}(\mathrm{II})$ ions by the Fen-ton reaction [21] to form reactive hydroxyl radical and life became very dangerous. Superoxide dismutase (SOD), the oldest enzyme that scavenges ROS effectively was found in all kingdoms of life; moreover, SOD has been evolved even before the eubacteria differentiation from archaea [8]. The GOE left many finger prints in the rocks and those records clearly show the early Earth's evolution [20]. The finding of old history of SOD strongly approves the simultaneous ROS appearance together with photosynthesis, dioxygen and first oxidative enzymes. Considering the current role of ROS in modern plants and mammals together with dioxygen activation mechanisms, one has to keep in mind that aerobic evolution on the Earth occurred in the presence of ROS and increasing $\mathrm{O}_{2}$ concentration. The first primitive organisms have finally evolved the strategy to survive and cope with the abundant dioxygen in the GOE-growing atmosphere. The $\mathrm{O}_{2}$ utilizing enzymes started to use paramagnetic metal ions, which provided spin-crossover in their $\mathrm{d}$-shell assisting the T-S transition in dioxygen moiety during re-dox reactions with substrates [1$3,14]$. This type of spin catalysis is rather simple and determined by exchange interactions [3]. Formally, catalysis by metal cofactor is allowed by spin-selection rules and its efficiency depends on the differences in exchange integrals for different electron pairs in the transition state region Otherwise, the evolved metal-free cofactors are more complicated in terms of their spin dynamic mechanisms. These spin transformations are met in numerous flavoenzymes $[2,19]$. Flavins and related pterins are extremely miscellaneous and versatile cofactors, which capable to activate $\mathrm{O}_{2}$ [18].

\section{Oxidases and Monooxygenases with Flavin Cofactors}

Now we want to consider the role of electron spin and internal magnetic interactions in the dioxygen open-shell, which are responsible for $\mathrm{O}_{2}$ activation by flavin-containing enzymes. This very wide class of catalysts provides numerous oxidation and oxygenation reactions in living cells. Some general results from such consideration will lead to useful conclusions about aerobic evolution and the role of dioxygen spin in our life.

Flavoenzymes as a widespread class of proteins are more adjusted to $\mathrm{O}_{2}$ activation than free flavins (Figure 1). Besides their enabling for efficient oxidations of the target substrates by triplet dioxygen they can supply the reduced reactive oxygen species to arrange signaling network system in the cell. ROS have been referred long time as "the double-edged sword of life" because of their toxic nature but al-so of their beneficial role [8]. The modern trends stress the great importance of the late role especially for plants $[8,18]$. Physical, chemical and biological principles by which flavoenzymes realize catalytic action upon dioxygen have been the subject of intense studies during last sixty years [5-7,18]. Current understanding of these rules is based mostly on seminal works of Vincent Massey [7,19]. A wealth of kinetic, mechanistic, computational and spectroscopy data [7] represent his consistent view on the functional and structural properties of flavin-containing oxidases and oxygenases. However, not all aspects of flavoproteincatalyzed bioreactions have been solved yet [18]. The major area 
of the flavoenzyme research still concentrates on elucidation of the electronic mechanisms and molecular basis for the dioxygen spin activation [1,22].

Nowadays, more than $10^{5}$ protein sequences deposited in the protein database are considered as flavin-dependent enzymes [18]. Flavoenzyme cofactors usually include either flavin adenine dinucleotide (FAD) or flavin mononucleotide (FMN), both being synthesized from vitamin B2 in vivo (Figure 1). The $\mathrm{O}_{2}$ spinactivating mechanism of flavoenzymes was first proposed for oxidases [2]. For example, glucose oxidase (GO) utilizes dioxygen as an electron acceptor in order to produce hydrogen peroxide [2-5]. Similar spin flip occurs when one oxygen atoms from $\mathrm{O}_{2}$ molecule is incorporating into the products; in this way monooxygenases activate dioxygen through spin catalysis by forming the $\mathrm{C}(4 \mathrm{a})$ peroxyflavin intermediate, which then reacts with substrate and inserts one oxygen atom into substrate by ordinary chemical rearrangement [18,22]. Free reduced flavins also react with dioxygen in solvents quite efficiently, forming oxidized flavin and hydrogen peroxide faster than in one second [19]. Stopped-flow spectra, EPR and rapid quenching techniques were used for such rapid reactions in order to understand their high rate in spite of the spin prohibition [7]. The oxidized flavin (Flox) was monitored by its strong absorption increase with a maximum at 450nm (Figure 1). The short delay in the beginning indicated a reaction intermediate formation. Addition of SOD inhibited the reaction; thus, the superoxide involvement became clear at the first stage of the process (Figure 2) [7].

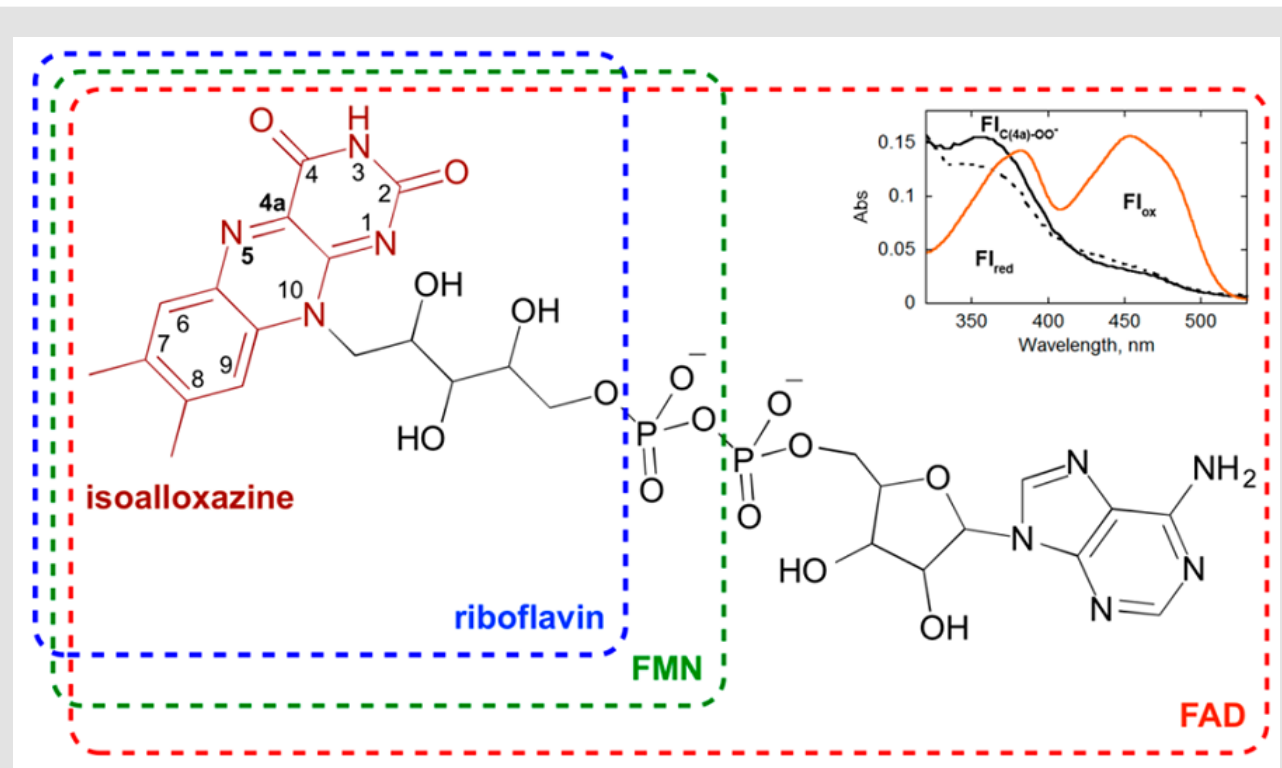

Figure 1: Structures of riboflavin (vitamin B2), FMN and FAD cofactors. Inset shows the UV-visible absorption spectra of the reduced (broken line) and oxidized flavin (red line), the C(4a)-peroxyflavin is presented by black line. From Ref. [18] with perimission.

This scheme was generalized later for flavoenzymes. The active center of enzyme is denoted in Figure 2 by square brackets. Electron transfer from reduced flavin (Flred) to $\mathrm{O}_{2}$ occurs as soon as dioxygen penetrates through protein channels into the active cite and occupies a close position to cofactor in the cavity between FAD and amino acid residues [1,2]. This result was supported first by density functional theory (DFT) calculations for the active center model of glucose oxidase [4]. Later on the similar mechanism was confirmed by numerous studies of a number of haem- and flavorenzymes, oxidoreductases, oxidases and oxygenases [22-44]. The radical pair between semiquinone (Flsq) and superoxide radicals $\left(\mathrm{O}_{2}-\bullet\right)$ in Figure 2 is originally generated in the triplet state because of the $\mathrm{O}_{2}$ triplet precursor. But the triplet state cannot produce products shown in Figure 2. From kinetic studies of reduced flavins
(Flred) reactions with dioxygen in solvents Vincent Massey have proposed the following reaction scheme [19]:

$$
\mathrm{Fl}_{\text {red }}(\uparrow \downarrow)+\mathrm{O}_{2}(\uparrow \uparrow) \rightarrow\left[\mathrm{Fl}_{s q} \cdot \uparrow \uparrow \mathrm{O}_{2}^{-\cdot}\right] \rightarrow\left[\mathrm{Fl}_{s q} \cdot \uparrow \downarrow \mathrm{O}_{2}^{-\cdot}\right] \rightarrow \mathrm{FlOO}^{-} \mathrm{FlOOH}(3)
$$

EPR spectra of semiquinones (Flsq) show a large spin density on $\mathrm{C}(4 \mathrm{a})$ atom [7]. The product was identified as the $\mathrm{C}(4 \mathrm{a})$-flavin hydroperoxide (Figure 2) by detection of the 450 and $370 \mathrm{~nm}$ absorption bands (Figure 1). In aqueous media this rather unstable product transforms heterolytically to the oxidized flavin (Flox) and $\mathrm{H}_{2} \mathrm{O}_{2}$ [19] (Figure 2). The late final product is typical for oxidase enzymes and intermediate flavinC(4a) hydroperoxide characterizes monooxygenases behavior (Figure 2) [23-51]. Thus, the mechanism of Eq. (3), proposed initially for reduced free flavins in solvents [19], was generalized for flavoenzymes (Figure 2) and postulates 
the following scenario. As in the solvent case, the electron transfer to $\mathrm{O}_{2}$ produces the caged triplet radical pair in the active cite of enzyme, which is capable (by unknown rea-sons) to undergo the $\mathrm{T} \rightarrow \mathrm{S}$ transition $[18,19]$. After such intersystem crossing, de-noted in Eq. (3), the normal singlet state chemistry follows and the singlet state radical pair proceeds to the short-lived Flo0- singlet species, which transforms into the intermediate $\mathrm{C}(4 \mathrm{a})$-flavin hydroperoxide (in monooxygenases) or into the hydrogen peroxide and oxidized flavin (Flox) in oxidases (Figure 2).

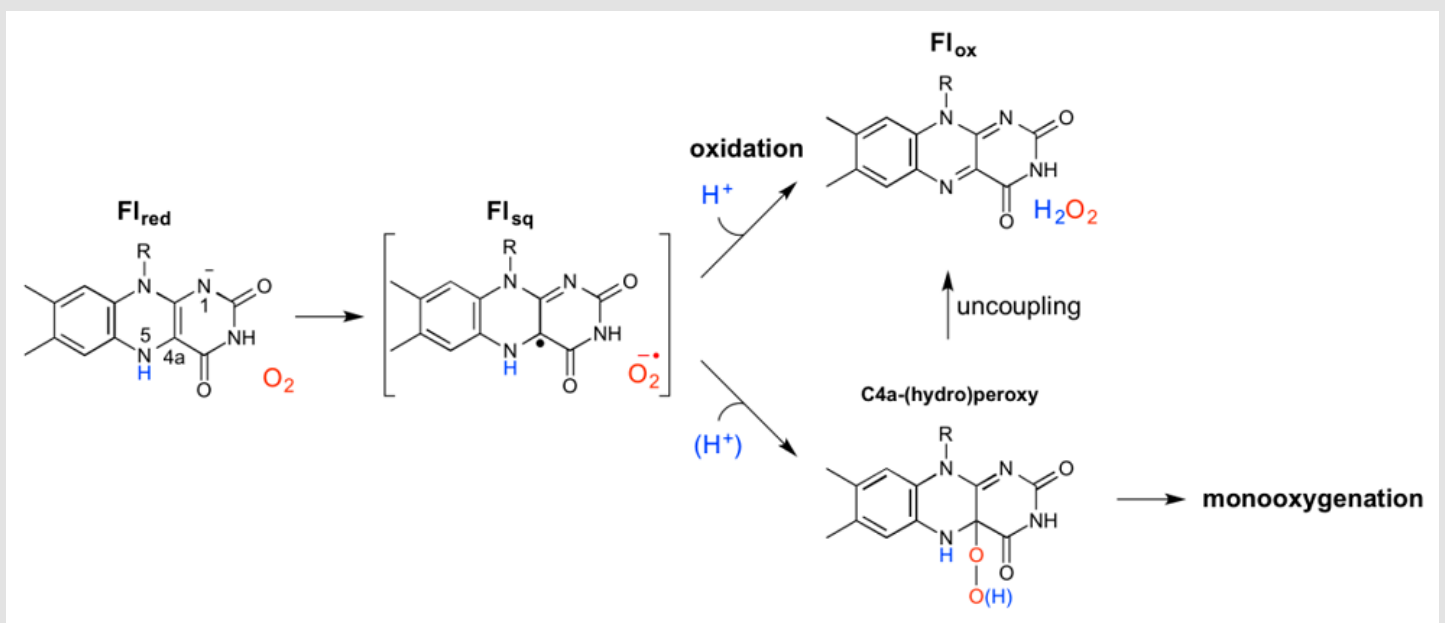

Figure 2: Typical dioxygen reactions catalized by oxidases and monooxygenases. Reactive atoms and positions are noted. From Ref. [18] with perimission.

Massey [19] and all his ancestors [20] accept the spin flip at the radical pair stage in Eq. (3) for granted (without any comments or explanations). One can propose that Massey has conceived the arguments of the well-known radical pair theory (RPT) based on the cage effect in solvent and account for hyperfine coupling with nuclear spins. RTP has been proposed 50 years ago [33] being very popular in nineties [19]. But, the RTP ideas cannot be applied to enzyme active center, where the radical pair is not separated in two radicals by a long distance ( $>10 \mathrm{~nm}$ like in the solvent cage) [21]. Instead of very weak hyperfine interactions between electron and nuclear spins, which cannot induce T-S transition in flavoenzymes by no means, one has to consider another driving force to provide the spin flip in reaction presented in Eq. (3). If we suppose that only thermodynamic parameters (like in traditional chemistry) govern chemical reactivity of $\mathrm{O}_{2}$ molecule, rather than the spin-dependent kinetics, then in this case all organic part of the Earth's biosphere would be consumed rapidly and converted into carbon dioxide and water; thus, aerobic life would be impossible. Life does exist because of specific internal magnetic interactions in the dioxygen open shell in a huge number of enzymes specified by Eq. (3).

\section{Spin-Orbit Coupling in Dioxygen Open Shell}

Now we have to consider $\mathrm{O}_{2}$ structure in more details. The dioxygen molecule in the triplet ground state $O_{2}\left(X^{3} \Sigma_{g}^{-}\right)$is characterized by the following electronic configuration $\left(1 \sigma_{\mathrm{g}}\right)^{2}$ $\left(1 \sigma_{\mathrm{u}}\right)^{2}\left(2 \sigma_{\mathrm{g}}\right)^{2}\left(2 \sigma_{\mathrm{u}}\right)^{2}\left(3 \sigma_{\mathrm{g}}\right)^{2}\left(1 \pi_{\mathrm{u}}\right)^{4}\left(1 \pi_{\mathrm{g}}\right)^{3}$ [9]. The first 14 electrons occupy 7 low-lying molecular orbitals (MO) by pairs with opposite spins. The $1 \pi \mathrm{u}$ and $1 \pi \mathrm{g}$ MO are doubly degenerate; thus, the two outer electrons have to be dis-posed in two degenerate $1 \pi \mathrm{g}-\mathrm{MOs}$. According to molecular analog of the Hund rule this implies to set two non-paired electrons with the same spin orientations. Such configuration provides the lowest triplet state of the type $[\uparrow][\uparrow]$, where the quantum cells denote two degenerate $1 \pi$ g-orbitals. The Hund rule and these two unpaired electrons in antibonding $1 \pi \mathrm{g}$ MOs are responsible for the specific character of dioxygen reactivity in combustion and in bio-oxidation. Two antibonding $1 \pi$ g-vacancies make possible to transform dioxygen into reactive species like superoxide $\mathrm{O}_{2}-\bullet$ and peroxide $\mathrm{O}_{2}{ }^{2}$ - anions. Next property of the $(1 \pi \mathrm{g})^{2}$ open shell determines probability to generate the other reactive species - singlet oxygen $\mathrm{O}_{2}(\mathrm{a} 1 \Delta \mathrm{g})$ [1]. One can construct four possible quantum states, Eqs. (4) and (5), for the electronic configuration $(1 \pi \mathrm{g})^{2}$. Let us consider cylindrical coordinate system, where $\theta$ is an angle between the radius vector (r) and the molecular axis ( $\mathrm{z}), \phi$ is the rotation angle of the radius vector about the $\mathrm{z}$ axis. In this coordinate system the wave functions for two degenerate $\pi g-M O$ 's have a form $|\pi \pm g\rangle=\psi(r, \theta)$ e $\pm i \phi$. They are eigenfunctions of the orbital angular momentum projection Lz on molecular $\mathrm{z}$ axis: $L_{z}\left|\pi_{g}^{ \pm}>= \pm \hbar\right| \pi_{g}^{ \pm}>$. Using this imaginary form of $\pi g$-MOs, the quantum states of $\mathrm{O}_{2}$ and $\mathrm{O}_{2}$ - molecule can be represented by the following scheme [1]:
$[\uparrow][\uparrow]$
$[\uparrow \downarrow][]$
[]$[\uparrow \downarrow]$
$[\uparrow][\downarrow]$
$[\uparrow \downarrow][\uparrow]$
$[\downarrow][\uparrow \downarrow]$
$O_{2}{ }^{3} \Sigma_{g}^{-} \quad O_{2}{ }^{1} \Delta_{g}$
$\mathrm{O}_{2}{ }^{1} \Delta_{g}$
$\mathrm{O}_{2}{ }^{1} \Sigma_{g}^{+}$ $\mathrm{O}_{2}^{-\bullet} \Pi_{g}$ 
These configurations of the $\pi \mathrm{g}$ open shell are also eigenfunctions of $L_{z}$ operator with eigenvalues $0, \pm 2 \hbar, 0$. Exchange interaction stabilizes the triplet state $\mid 3 \mathrm{Sg}->$ as the lowest one; the degenerate singlet states $\mid 1 \Delta \mathrm{g}>$ are higher in energy by $22 \mathrm{kcal} / \mathrm{mol}$, while the $\mid 1 \mathrm{gg}+>$ state is the highest one with a total energy $36 \mathrm{kcal} / \mathrm{mol}$ [9]. Wave functions of these states in the form of Slater determinants are:

$$
\begin{aligned}
& { }^{1} \Delta=\left|\pi^{+} \bar{\pi}^{+}\right| \quad{ }^{1} \Delta=\left|\pi^{-} \bar{\pi}^{-}\right| \\
& { }^{3} \Sigma^{-}=\frac{1}{\sqrt{2}}\left(\left|\pi^{+} \bar{\pi}^{-}\right|-\left|\pi^{-} \bar{\pi}^{+}\right|\right) ; \quad{ }^{1} \Sigma^{+}=\frac{1}{\sqrt{2}}\left(\left|\pi^{+} \bar{\pi}^{-}\right|+\left|\pi^{-} \bar{\pi}^{+}\right|\right) ;
\end{aligned}
$$

Some symbols are omitted here and only two-electronic parts of wave functions are shown [1]. Spin orbit coupling (SOC) between $\mathrm{S}$ and $\mathrm{L}$ angular momenta is known to be responsible for magnetic spitting of orbitally degenerate spin multiplets in atoms and diatomic molecules [9]. For diatomics such splitting is possible for states with the wave functions being eigenfunctions of the $L_{z}$ operator $\left(L_{z} \Psi=\Lambda \hbar \Psi\right)$, for which $\Lambda \neq 0$ [9]. Naturally, magnetic spitting indused by SOC is possible only for states with spin quantum number $S \neq 0$ [9]. In such case the spin and orbital angular momenta projections on the molecular axis are observable values and their coupling depends on mutual orientation of both magnetic moments. This provides a well-seen SOC-induced splitting of the molecular $\Pi, \Delta, \Phi$ multiplets (typically of the order $0.01 \mathrm{eV}$ in light molecules) [36]. In this work we use a simple semiempirical approximation for spin-orbit coupling (SOC) operator [10]

$$
H_{s o}=\sum_{A} \zeta_{A} \sum_{i} l_{i, A} . S_{i}=\sum_{i} B_{i} . S_{i}
$$

Here $\zeta A$ - is a spin-orbit coupling constant for the valence shell of atom A, deter-mined from atomic splitting, - are the orbital and spin angular momenta operators (in $\hbar$ units) for the $i$-th electron [1]. Expectation value of this operator is equal zero for the ground triplet state $\mathrm{X}^{3} \Sigma_{\mathrm{g}}$ - of the $\mathrm{O}_{2}$ molecule, since it has $\Lambda=0$. But the $\mathrm{S}_{\mathrm{z}}$ $=0$ component of the triplet state, shown in Eq. (5), is still slightly split from the upper $S_{z}= \pm \hbar$ components in the second order of perturbation theory, $\Delta \mathrm{E}(2)$, since the triplet and singlet states in Eq. (5) are mixed by spin-orbit coupling matrix element [11]:

$$
\left\langle X^{3} \sum_{g, 0}^{-}\left|H_{S O}\right| b^{1} \sum_{g}^{+}\right\rangle<\pi_{x}\left|B_{z}\right| \pi_{y}>\zeta_{0}=0.022 e v
$$

This integral is reduced to the spin-orbit coupling constant for the atomic oxygen $\mathrm{O}(3 \mathrm{P})$ ground state and this is the largest possible energy of magnetic internal interaction in all oxygen all atrops. In the absence of external magnetic field the spitting between spin sublevels $\mathrm{ms}=0$ and $\mathrm{ms}= \pm 1$ of the ground triplet state $\mathrm{X}^{3} \Sigma_{\mathrm{g}}$ - of the $\mathrm{O}_{2}$ molecule, produced by SOC contribution from Eq. (7) [10] is equal to:

$$
\Delta \mathrm{E}^{(2)}=\left|<X^{3} \sum_{g, 0}^{-}\right| H_{S O}\left|b^{-1} \sum_{g}^{+}>\right|^{2}\left[E\left(b^{-1} \sum_{g}^{+}\right)-E\left(X^{3} \sum_{g}^{-}\right)\right]^{-1}=0.0003 e v
$$

Here the S-T energy gap is equal $1.63 \mathrm{eV}$ (from experiment [9]). The calculated $\Delta \mathrm{E}(2)$ spitting is equivalent to $2.42 \mathrm{~cm}^{-1}$, which provides $61 \%$ of the total observed zero- field splitting (ZFS=3.98 $\mathrm{cm}^{-1}$ ) [10]. The rest is determined by direct spin-spin coupling of two electrons [45-47]. This rather small measure of internal magnetic forces in the dioxygen ground triplet state have no direct connection with the origin of $\mathrm{O}_{2}$ activation by enzymes. However, it is important for the singlet dioxygen excitation and quenching in solvents [11-13]. Account of Eq. (7) in the first order of perturbation theory provides a mixture of two states from Eq. (5).

$$
\begin{aligned}
& \Psi_{b}=\left|\tilde{\emptyset} \Sigma_{g}{ }^{+}>+c\right|{ }^{3} \Sigma_{g, 0}{ }^{-}> \\
& \Psi_{X, 0}=\left|\tilde{O}^{3} \Sigma_{g, 0}{ }^{-}>-c^{*}\right| b^{1} \Sigma_{g}{ }^{+}>
\end{aligned}
$$

where coefficient is equal

$$
c=\frac{\left\langle X^{3} \sum_{g, 0}^{-}\left|H_{S O}\right| b^{-1} \sum_{g}^{+}\right\rangle}{E\left(b^{-1} \sum_{g}^{+}\right)-E\left(X^{3} \sum_{g, 0}^{-}\right)}
$$

$=0.0135$. This small co-efficient of magnetic admixture between ground triplet and excited singlet states is very important, being responsible for many peculiarities of dioxygen [1]. It explains a magnetic transition moment for $\mathrm{X} \rightarrow \mathrm{b}$ absorption and intensity of the famous Fraunhofer line at 760nm in the Sun spectrum [15], intensity borrowing scheme for $\mathrm{a}-\mathrm{X}$ transition from the Noxon $\mathrm{a}-\mathrm{b}$ band in solvents and many others optical $\mathrm{O}_{2}$ effects [1]. Thus, the SOC integral, Eq. (7), plays a great role in the theory of oxidative stress and toxicity of reactive oxygen species [15]. The dioxygen activation by flavoenzymes in our spin-magnetic treatment means such mechanistic pathways that cause the release of spin prohibition in $\mathrm{O}_{2}$ reactions with diamagnetic substrates, Eq. (1), making them effectively allowed [2]. It is important that $\mathrm{T}$ and $\mathrm{S}$ wave functions in Eq. (5) differ by electron rotation around z-axis; this creates appearance of orbital angular momentum during the T-S transition, which in turn simultaneously provides a torque to realize the spin flip [22]. The similar quantum-mechanical ideas can be applied to explain mysterious spin flip in Eq. (3) of Vincent Massey [2,22]. Let us consider electronic configuration of superoxide ion $\mathrm{O}_{2}-\bullet$ by addition one more electron to dioxygen: $(1 \sigma g)^{2}(1 \sigma u)^{2}(2 \sigma g)^{2}(2 \sigma u)^{2}$ $(3 \sigma g)^{2}(1 \pi u)^{4}(1 \pi g)^{3}[2]$. For such $(1 \pi g)^{3}$ open shell in the absence of SOC account we have two degenerate configurations (a) and (b) with $m s=1 / 2$, and similar two $\left(\mathrm{a}^{*}, \mathrm{~b}^{*}\right)$ - for $\mathrm{ms}=-1 / 2$.

$$
\begin{array}{llll}
{[\uparrow \downarrow][\uparrow]} & {[\uparrow][\uparrow \downarrow]} & {[\uparrow \downarrow][\downarrow]} & {[\downarrow][\uparrow \downarrow]} \\
{ }^{2} \Pi_{3 / 2}(a) & { }^{2} \Pi_{1 / 2}(b) & { }^{2} \Pi_{1 / 2}\left(a^{*}\right) & { }^{2} \Pi_{3 / 2}\left(b^{*}\right)(10)
\end{array}
$$

With account of spin-orbit coupling, Eq. (6), in the first order of perturbation theory these four configurations will be spit in two sublevels: $\mathrm{E}\left({ }^{2} \Pi_{1 / 2}\right)-\mathrm{E}\left({ }^{2} \Pi_{3 / 2}\right)=\zeta O$ [2]. This is in a good agreement with experimental result for $\mathrm{O}_{2}-\bullet$ ion: $E\left({ }^{2} \Pi_{1 / 2}\right)-E\left({ }^{2} \Pi_{3 / 2}\right)=160$ $\mathrm{cm}^{-1}(0.02 \mathrm{eV})$ [48]. Such energy is relatively large for SOC in light molecule $[49,50]$ and is determined by the observable orbital angular momentum of the ${ }^{2} \Pi$ state $\left(L_{z}=\hbar\right)$. It is million times larger than a typical hyperfine coupling used in the radical pair theory to induce T-S flip [33]. The same type of orbital rotation around $\mathrm{z}$ axis, 
as in Eq. (7), determines this internal magnetic energy. The splitting is inverted $\left(\Omega=3 / 2\right.$ is lower than $\Omega=1 / 2$ sublevel), since the $(1 \pi \mathrm{g})^{3}$ open shell is more-than-half occupied [14]. Here $\Omega$ is the total angular momentum projection $\Omega=\mid \Lambda+$ Sz $\mid$ [9]. Now we can apply this result to the Massey's mechanism of Eq. (3) [19], including our quantum cell notation [] for molecular orbitals and the curly brackets for the enzyme active center:

$$
\begin{aligned}
& F l_{\text {red }}[\uparrow \downarrow]+O_{2}[\uparrow][\uparrow] \rightarrow\left\{F l_{s q} \cdot[\uparrow] \ldots[\uparrow \downarrow][\uparrow] O_{2}^{-*}\right\} \underline{\operatorname{SOC}}\left[F l_{s q} \cdot[\uparrow] \ldots[\downarrow][\uparrow \downarrow] O_{2}^{-*}\right] \\
& \text { (a) } \\
& \rightarrow \text { FlOO }^{-}[\uparrow \downarrow][\uparrow \downarrow]+H^{+} \text {FlOOH }[\uparrow \downarrow][\uparrow \downarrow] \\
& \text { (b*) }
\end{aligned}
$$

The triplet $\rightarrow$ singlet quantum transition in the enzyme active center includes entirely the (a) $\rightarrow\left(b^{*}\right)$ spin flip induced by SOC inside the superoxide moiety $[2,4]$. The unpaired spin in flavin semiquinone $\left(\mathrm{F}_{\mathrm{lsq}}\right)$ is inactive in the T-S transition, Eq. (11). Molecular orbitals of the superoxide ion in the enzyme cage are perturbed by protein scaffold and by cofactor; thus they are not completely degenerate [4]. But molecular $\mathrm{O}_{2}-\bullet$ ion can rotate inside the cavity; this hindered rotation will change the positions of the ${ }^{2} \Pi_{1 / 2}$ and ${ }^{2} \Pi_{3 / 2}$ sublevels and their splitting. Energies of all four configurations (10) could be determined mostly by electrostatic perturbations rather than by SOC [14]. This asymmetric top will change the sublevels splitting and order with the frequency of libration movement. Thus, the energies of the (a) and ( $\left.b^{*}\right)$ configurations would be close to resonance periodically in the picoseconds time scale. The same is true for the (b) and (a*) configurations in Eqs. (10)-(11). The most important is the fact that spin-orbit coupling between such $\mathrm{T}$ and

$S$ states is always large and close to $(1 / 2) \varsigma_{O}$, since the structure of two $\pi \mathrm{g}-\mathrm{MO}$ 's are not changed much [1]. In superoxide ion they are similar to $\pi \mathrm{g}, \mathrm{x}$ and $\pi \mathrm{g}, \mathrm{y}$ orbitals of $\mathrm{O}_{2}$ molecule and their structure is not perturbed much in the enzyme active center. Small admixtures from the nearest neighbors do not change the orbital torque between (a) and ( $b^{*}$ ) configuration in the scheme, Eq. (11), which remains similar to the orbital rotation in Eq. (7). The (1/2) coefficient is the only main difference between SOC matrix element in scheme of Eq. (11) and in Eq. (7). This factor ( $1 / 2$ ) always enters the T-S matrix elements of SOC operator, but Eq. (7) is a particular exclusion because of specific structure of wave func-tions (5) [10].

The $\mathrm{T}$ and S configurations of the (a) and $\left(\mathrm{b}^{*}\right)$ types have been first taken into account for explanation of the charge-transfer contributions in SOC enhancement during the singlet oxygen quenching by aliphatic amines [49]. In the triplet oxygen activation by flavins and flavoenzymes presented in Eq. (11) this SOC effect is realized in the most simple and clear form $[1-4,49]$. The particular electronic structure of the superoxide ion, Eq. (10), provides very efficient magnetic torque from $\pi \mathrm{g}$ orbital rotation, which induces by SOC operator the spin flip during T-S transition. The twisting force that tends to cause spin rotation in the mechanism described by Eq. (11) is unusually strong for such light molecule as dioxygen. That is why the Massey's mechanism is so efficient in many enzymes [22-30]. The T-S matrix element of SOC operator in enzyme active center described by Eq. (11) is equal to $\mathrm{i}\left(1 \frac{1}{2}\right) \zeta \mathrm{O}=\mathrm{i} 0.01 \mathrm{eV}$ being imaginary value [2]. Calculation of the $\mathrm{T} \rightarrow \mathrm{S}$ transition rate constant includes the square of this absolute value [4]; thus, the imaginary unit $(i=\sqrt{ }-1)$ does not matter for the measurement of rate constant and can be omitted. The SOC energy of $10^{-2} \mathrm{eV}$ realized inside small $\mathrm{O}_{2}-\bullet$ species (about $0.2 \mathrm{~nm}$ diameter) corresponds to magnetic force of about $10^{-6}$ dyne. This is relatively strong force for quantum microparticles. We mean the force exerted between spin magnet and orbital movement ( $\pi \mathrm{g}, \mathrm{x} \rightarrow \pi \mathrm{g}$,y rotation) of electrically charged particle (electron). Such force is much stronger than those magnetic forces, which deter-mine rate of T-S transitions in phosphorescence of organic molecules [50]. The rate constant for T-S transition in the caged radical pair of flavoenzyme shown in scheme of Eq. (11) can be in the range $10^{4}-10^{6} \mathrm{~s}^{-1}[12,39]$. Such fast intersystem crossing rate can compete with other possible relaxation rate processes at the elec-tron transfer stage in the enzyme active center, Eq. (11) $[12-17,51]$. Decay of the radical pair and the superoxide leakage from the cage of enzyme are slow processes hindered by the tight protein scafold (though neither flavin nor dioxygen provide covalent binding with nearest amino acid resudues) [5-7]. When the reduced oxidases react with dioxygen and form $\mathrm{H}_{2} \mathrm{O}_{2}$ these reactions have been investigated by stopped-flow techniques for a number of flavoenzymes, like GO, cholesterol, alditol and sarcosine oxidases, p-hydroxyphenyl acetate hydroxylase [7,19]. The measured rate constants demonstrate pseudo-first-order character being linear in dioxygen concentration with effective bimolecular rate constant of the order $10^{6}-10^{7}$ mole- $1 \mathrm{~s}^{-1}[18,19]$. These reactions are much faster than $\mathrm{O}_{2}$ reactions with free flavins reduced in solvents with rate constants about 250 mole $^{-1} \mathrm{~s}^{-1}[5,18]$. This means that protein environment of enzyme not only as-sists in the electron transfer to $\mathrm{O}_{2}$ [4], but also enhances vibrational maintenance of intersystem crossing process [39].

\section{Cofactor-Independent Oxygenation}

From the mechanism of Eq. (11) it is clear that flavin cofactor does not contribute to the SOC matrix element $<\pi_{g, x}(a)\left|H_{s o}\right| \pi_{g, y}\left(b^{*}\right)>$, since the nonpaired electron spin of flavin semiquinone (Flsq) plays only role of a passive spectator [17]. The role of Flavin cofactor is to provide electron transfer for $\mathrm{O}_{2}$ eduction and to arrange proper binding of intermediates in the course of reaction pathway on the singlet state potential energy surface. The redox properties of Flavin are the main factor of their enzyme activity. If the organic substrate possesses similar redox potentials it can himself activate dioxygen for the spin flip without cofactor assistance [17]. Such cofactor-free enzymes are really discovered during last decade [34,35,52-54] and considered as a new paradox of dioxygen activation [55]. In this respect, an example of the bacterial 2,4-dioxygenase from Arthrobacter nitro guajacolicus Ru61a, which operates without any cofactor and can 
efficiently degrade quinolones [52], attracts much theoretical attention [17,22,27,31,34]. This 1-H-3-hydroxy-4-oxoquinaldine 2,4-dioxygenase (enzyme named as HOD) [33] is capable to decompose 2-alkyl-3-hydroxy-4(1H)-quinolone (AHQ) substrate by dioxygen and produce $\mathrm{N}$-acetyl-anthranilate $+\mathrm{CO}$ products [52]. On the ground of DFT calculations Hernández-Ortega, et al. [31] have proposed a new mechanism based on primary triplet diradical intermediate $3 \mathrm{I} 1$ produced by dioxygen covalent binding with substrate at 2-position. The authors of Ref. [31] assumed (without SOC calculations) that T-S transition occurs at the peroxyl diradical 3 I1 stage with subsequent intersystem crossing to the singlet 1I1 peroxide inter-mediate followed by usual chemical decomposition of quinolone to anthranilate at the 1I2 stage with typical decarboxylation.

Other authors [17,22,39] have shown that SOC calculation cannot approve such intersystem crossing mechanism since the covalently bound 3I1 peroxyl diradical has no enough internal magnetic force to induce efficient spin flip; orbital rotation is quenched by $\mathrm{O}_{2}$ chemical binding at the $3 \mathrm{I} 1$ stage [22]. At the same time the electron transfer and superoxide formation are possible at the first stage of HOD enzyme catalysis [27]. Redox properties of quinolone AHQ afford such electron jump from deprotonated substrate anion to $\mathrm{O}_{2}$. In this case the efficient SOC in the triplet radical pair between $\mathrm{O}_{2}-\bullet$ and deprotonated substrate radical is emerged by the same mechanism as in the flavoenzymes catalysis, Eq. (11), since such spin-orbit coupling is determined entirely by the $\pi \mathrm{g}$ open shell of superoxide ion [22]. The spin flip (T-S transition) induced by efficient SOC does happen and the simple chemical transformations proceed further on the singlet state potential energy surfaces according to calculation scheme presented in Ref. [31]. The main feature of this catalysis by HOD and by other cofactor-free enzymes [55] is that their substrates are capable for deprotonation; thus, substrate anion himself can catalyze an electron transfer to $\mathrm{O}_{2}$ and generate substrate radical and superoxide ion radical pair in the initial triplet state. Then our mechanism of internal magnetic interaction inside $\mathrm{O}_{2}^{-} \bullet$ moiety, shown in Eq. (11), can be applied for all cofactor-free enzymes of similar type [35,55]. Internal magnetic property of the superoxide open shell, Eq. (10), provides orbital torque, which is responsible for T-S transition in such radical pair [2]. After this spin flip the singlet state with paired spins can recombine to form a hydroperoxide intermediate followed by other singlet state reactions toward selective products $[34,35]$. The main driving force for all considered oxidation and oxygenation processes is a magnetic torque created by the $(a) \rightarrow\left(b^{*}\right)$ electron jump with simultaneous spin flip, shown in Eq. (11). Dioxygen activation by all considered enzymes [2244,51-56] starts with the triplet radical pair formation which includes superoxide ion. Spin-orbit coupling in the $\mathrm{O}_{2}-\bullet$ moiety, Eq. (10), provides effective removal of spin prohibition through the high rate of intersystem crossing (T-S transition) in such radical pair [1-4]. All biochemists must be convinced in this mechanism of $\mathrm{O}_{2}$ activation since the strength of magnetic force responsible for the spin flip is proved by experimental measurement of the spin splitting in the ground doublet state $\mathrm{X}^{2} \Pi_{\mathrm{g}}$ of superoxide ion [48].

\section{Conclusion}

Oxidation of organic matter by the triplet $\mathrm{O}_{2}$ molecule from the air (dioxygen) is a spin-forbidden process in the absence of ignition by radicals. Reactivity of dioxygen starts to attract a new interest recently by few reasons. This is a challenge for basic researches, which aim to highlight the most fundamental aspects of dioxygen chemistry being not solved since the Lavoisier and Faraday discoveries. The former has shown that $\mathrm{O}_{2}$ gas is responsible for respiration and combustion; the late - determined para magnetism of dioxygen. It became clear now that only modern quantum chemistry at the level of relativistic theory can approach to solution of the metabolic oxygenation and respiration problems. In 2002 the author of this review has proposed a general answer to the question "How enzymes can activate the triplet ground state dioxygen for organic substrates oxidation?" [2] The answer reduces this complicated biological problem to analysis of weak internal magnetic perturbations in the active sites of two enzymes, glucose oxidase and copper amine oxidase [2]. A number of recent publications support these ideas and extend their applications to various enzymes. One can see that reduced flavin in the singlet ground state initially reacts with the triplet dioxygen by a single electron transfer, followed by the rapid T-S transition with subsequent formation and decay of a covalently bound intermediate. A common characteristic of the oxygen-utilizing flavoenzymes is the dramatic rate increase in comparison with free flavins for the triplet dioxygen reduction. Some monooxygenases are able to produce flavin hydroperoxide as a metastable intermediate and then to use this reactive species in various useful synthesis as nucleophile or electrophile reagents. With new knowledge of magnetic physical mechanisms of dioxygen activation these could be useful synthetic targets for drug design with high medical selectivity and special purposes.

All known flavoenzymes able to catalyze the triplet $\mathrm{O}_{2}$ reactions with organic substrates not only accelerate the rates of those reaction, but also govern the reactions along particular reaction paths, which lead to selective products [49-54]. This is determined by fast electron transfer and by T-S spin flip assisted by special organization of the nearest protein environment. DFT calculations show that some amino acid residues assist to provide electron transfer to dioxygen and influence spin-orbit coupling effect on $\mathrm{T} \rightarrow \mathrm{S}$ transition in the enzyme active center. This was shown first for oxidation half reaction in glucose oxidase from Aspergillus Niger [4] and latter supported by DFT calculations for many other enzymes 
[22-44]. The rate of spin flip strongly depends on protein scaffold structure as well as the subsequent chemical reactions along the singlet pathways.

Advent of photosynthesis and Oxygen Catastrophe in the first half of the Earth's billion-years history shacked the world and life evolution in such a way that eukaryotes got accustomed to cope with $\mathrm{O}_{2}$ in the air and developed various enzymes including superoxide dismutase [56]. Metal containing enzymes are able to overcome spin prohibition for $\mathrm{O}_{2}$ reactions with organic matter by simple spin-catalysis based on exchange interaction. The late has a natural origin being typical for chemical bonding, which, by the way, explains an easy oxidation of metals by air. The most difficult (from the mechanistic point of view) and unusual for traditional chemistry laws is a wide type of enzymes, which do not contain metals. The crucial chemical role in these enzymes plays a weak magnetic force in-side the superoxide $\pi g$ open shell, Eq. (10), which induces the $\mathrm{T} \rightarrow \mathrm{S}$ quantum transition and abolishes the severe spin prohibition on dioxygen reactivity. The unfavorable kinetic barrier for organic oxidation by the triplet $\mathrm{O}_{2}$ molecule is relaxed in the enzyme active center by one-electron reduction to $\mathrm{O}_{2}-\bullet$, which bears inside it-self a crucial magnetic force to induce the necessary spin flip leading to selective products without damage for the cellular milleu. One can say that this is the main driving force of all aerobic evolution on the Earth.

\section{References}

1. Minaev BF (2007) Electronic mechanisms of molecular oxygen activation. Rus Chem Reviews 76(11): 988-1010.

2. Minaev BF (2002) Spin effects in reductive activation of $\mathrm{O}_{2}$ by oxydase enzymes. RIKEN Reviw 44: 147-149.

3. Prabhakar R, Siegbahn PEM, Minaev BF (2003) A Theoretical Study of the Dioxygen Activation by Glucose Oxidase and Copper Amine Oxidase. Biochim Biophys Acta 1647(1-2): 173-178.

4. Prabhakar R, Siegbahn PEM, Minaev BF, Agren H (2002) Activation of triplet dioxygen by glucose oxidase: Spin-orbit coupling in the superoxide ion. J Phys Chem B 106: 3742-3750.

5. Klinmann JP (2001) Life as Aerobes: Are There Simple Rules for Activation of Dioxygen by Enzymes? J Biol Inorg Chem 6(1): 1-13.

6. Klinman JP (2007) How Do Enzymes Activate Oxygen without Inactivating Themselves? Acc Chem Res 40(5): 325-333.

7. Massey V (2002) The Reactivity of Oxygen with Flavoproteins. Int Congr Ser 1233.

8. Mittler R (2017) ROS are good. Trends in Plant Sci 22(1): 11-18.

9. Herzberg G (1950) Spectra of diatomic molecules. Van Nostrand Reinold Co.

10. Minaev BF (1978) Effect of spin-orbit coupling on the intensity of magnetic dipole transitions in molecular oxygen. Soviet Phys J 21(9): 1205-1209.

11. Minaev BF (1985) Theory of the solvent effect on the radiative probability of the a $1 \Delta \mathrm{g}-\mathrm{X}^{3} \mathrm{Sg}$ - transition in the oxygen molecule. Optics and Spectroscopy 58: 761-763.
12. Minaev BF (2017) Spin-orbit coupling mechanism of singlet oxygen $\mathrm{a} 1 \Delta \mathrm{g}$ quenching by solvent vibrations. Chem Phys 483-484: 25-46.

13. Bregnhøj M, Westberg M, Minaev BF, Ogilby PR (2017) Singlet oxygen photophysics in liquid solvents: converging on a unified picture. Acc Chem Res 50(8): 1920-1927.

14. Minaev BF (2009) Spin-catalysis in the processes of photo- and bioactivation of molecular oxygen. Ukr Biochem J 81(3): 21-45.

15. Minaev BF (2016) Photochemistry and spectroscopy of singlet oxygen in solvents. Recent advances which support the old theory. Chem \& Chem Technol 10(4): 519-530.

16. Minaev BF, Kobzev GI (2003) Response calculations of electronic and vibrational transitions in molecular oxygen induced by interaction with noble gases. Spectrochim Acta A 59(14): 3387- 3410.

17. Minaev BF, Panchenko AA (2019) Spin catalysis of Unsaturated Substrates Oxidation by Cofactor free Mono and Di oxygenases. How Triplet Oxygen Can Overcome Spin Prohibition. Ukr J Medicine Biology and Sport 4: 329-343.

18. Romero E, Castellanos JRZ, Giovanni Gadda G, Marco W Fraaije MW, Mattevi A (2018) Same Substrate, Many Reactions: Oxygen Activation in Flavoenzymes. Chem Rev 118(4): 1742-1769.

19. Massey V (1994) Activation of Molecular Oxygen by Flavins and Flavoproteins. J Biol Chem 269(36): 22459-22462.

20. Lyons TW, Reihard CT, Planavsky NJ (2014) The rise of oxygen in Earth's early ocean and atmosphere. Nature 506(7488): 307-315.

21. Zakharov II, Kudjukov KY, Bondar VV, Tyupalo NF, Minaev BF (2011) DFT-based thermodynamics of fenton reactions rejects the 'pure'aquacomplex models. Comput Theor Chemistry 964(1-3): 94-99.

22. Minaev BF (2019) How cofactor-free oxygenases can overcome spin prohibition in substrates oxygenation by dioxygen. Chem Phys 521: 6168.

23. Wongnate T, Surawatanawong P, Visitsatthawong S, Jeerus Sucharitakul, Nigel S Scrutton, et al. (2014) Proton-Coupled Electron Transfer and Adduct Configuration Are Important for C4a-Hydroperoxyflavin Formation and Stabilization in a Flavoenzyme. J Am Chem Soc 136(1): 241-253.

24.Wang XL, Quan JM (2011) Intermediate-Assisted Multifunctional Catalysis in the Conversion of Flavin to 5,6-Dimethylbenzimidazole by BluB: A Density Functional Theory Study. J Am Chem Soc 133(11): 40794091.

25. Kiss DJ, Ferenczy GG (2019) A detailed mechanism of the oxidative halfreaction of $\mathrm{D}$-amino acid oxidase: another route for flavin oxidation. Org Biomol Chem 17(34): 7973-7984.

26. Kannappan B, Cummins PL, Gready JE (2019) Mechanism of Oxygenase Pathway Reactions Catalysed by Rubisco from Large Scale Kohn-Sham Density Functional Calculations. J Phys Chem B 123(13): 2833-2843.

27. Silva PJ (2016) Refining the reaction mechanism of $\mathrm{O}_{2}$ towards its cosubstrate in cofactor-free dioxygenases. Peer J 4: e2805.

28. Silva PJ, Ramos MJ (2008) A comparative density-functional study of the reaction mechanism of the $\mathrm{O}_{2}$-dependent coproporphyrinogen III oxidase. Bioorganic Medicin Chem 16: 2726-2733.

29. Ortega P, Zanchet A, Sanz Sanz C, Gómez Carrasco S, Lola González Sánchez, et al. (2020) Dpg C-catalyzed peroxidation of DPA-CoA: insights into the spin-forbidden transition and charge transfer mechanisms. Chem Rxiv 27(5): 1700-1712.

30. Visitsatthawong S, Chenprakhon P, Chaiyen P, Panida Surawatanawong (2015) Mechanism of Oxygen Activation in a Flavin-Dependent Monooxygenase: A Nearly Barrierless Formation of C4a- 
Hydroperoxyflavin Via Proton-Coupled Electron Transfer. J Am Chem Soc 137(29): 9363-9374.

31. Hernández Ortega A, Quesne MG, Bui S, Roberto A Steiner, Nigel S Scrutton, et al. (2015) Catalytic mechanism of cofactor-free dioxygenases and how they circumvent spin-forbidden oxygenation of their substrates. J Am Chem Soc 137(23): 7474-7487.

32. Bathelliera C, Yuc LJ, Farquhar GD, Michelle L Coote, George H Lorimer, et al. (2020) Ribulose 1,5-bisphosphate carboxylase/oxygenase activates $\mathrm{O}_{2}$ by electron transfer. PNAS Latest Articles 117(39): 24234-24242.

33. Hore PJ, Mouritsen H (2016) The Radical-Pair Mechanism of Magnetoreception. Annual Review of Biophysics 45: 299-344.

34. Fetzner S, Steiner RS (2010) Cofactor-independent oxidases and oxygenases. Appl Microbiol Biotechnol 86(3): 791-804.

35. Baas BJ, Poddar H, Geertsema EM, Rozeboom HJ, Poelarends GJ, et al. (2019) Functional and Structural Characterization of an Unusual Cofactor-Independent Oxygenase. Biochemistry 50(5): 2889-2899.

36. Minaev BF, Minaeva VA (2008) Spin-dependent binding of dioxygen to heme and charge-transfer mechanism of spin-orbit coupling enhancement. Ukr Bioorg Acta 6(2): 56-64.

37. Minaev BF, H Ågren H, Minaeva V (2016) Spin-orbit coupling in enzymatic reactions and the role of spin in biochemistry. In: J Leszczynski (Edt.). Handbook of Computational Chemistry, Springer, Netherlands, p. 1-31.

38. Usselman RJ, Hill I, Singel DJ, Martino CF (2014) Spin Biochemistry Modulates Reactive Oxygen Species (ROS) Production by Radio Frequency Magnetic Fields. PLoS ONE 9: e93065.

39. Minaev BF, Valiev RR (2019) Spin-orbit coupling effects in $O_{2}$ activation by cofactor-independent 2,4-dioxygenase. Ukr Biochem J 91(4): 38-46.

40. Saito K, Watabe Y, Miyazaki T (2020) Spin-inversion mechanisms in 02 binding to a model heme compound: A perspective from nonadiabatic wave packet calculations. J Comput Chem 41(29): 2527-2537.

41. Kussmaul L, Hirst J (2006) The mechanism of superoxide production by NADH: ubiquinone oxidoreductase (complex I) from bovine heart mitochondria. PNAS 103(20): 7607-7612.

42. Minaev BF, Minaeva VA, Leschenko VN (2004) Electronic structure models of flavoproteides and mechanism of oxidases action. Biopolymers and Cell 20(3): 224-232.

ISSN: 2574-1241

DOI: 10.26717/BJSTR.2021.38.006171

Boris Minaev. Biomed J Sci \& Tech Res

(C) (P) This work is licensed under Creative Commons Attribution 4.0 License

Submission Link: https://biomedres.us/submit-manuscript.php
43. Roth JP, Klinman JP (2003) Catalysis of electron transfer during activation of $\mathrm{O}_{2}$ by the flavoprotein glucose oxidase. PNAS 100(1): 62-67.

44. Cho KB, Chen H, Janardanan D, Sam P de Visser, Sason Shaik, et al. (2012) Nonheme iron-oxo and -superoxo reactivities: $\mathrm{O}_{2}$ binding and spin inversion probability matter. Chem Commun 48(16): 2189-2191.

45. Vahtras O, Loboda O, Minaev B, Hans Agren, Kenneth Ruud (2002) Ab initio calculations of zero-field splitting parameters. Chem Phys 279(23): 133-142.

46. Minaev BF, Murugan NA, Ågren H (2013) Dioxygen spectra and bioactivation. Inter J Quant Chem 113(14): 1847-1867.

47. Minaev BF (2004) Ab initio study of the ground state properties of molecular oxygen. Spectrochim Acta A 60(5): 1027-1041.

48. Land JE, Raith W (1973) Fine Structure of $\mathrm{O}_{2}$ - Measured by Electron Time-of-Flight Spectroscopy. Phys Rev Lett 30: 349-352.

49. Minaev BF (1984) Spin-orbit coupling of charge-transfer states and the mechanism for quenching singlet oxygen by amines. Theor Experimental Chem 20: 199-201.

50. Baryshnikov G, Minaev B, Ågren H (2017) Theory and calculation of phosphorescence phenomena. Chem Rev 117(9): 6500-6537.

51. Minaev BF (2010) Environment friendly spin-catalysis for dioxygen activation. Chem \& Chem Technol 4(1): 1-16.

52. Frerichs Deeken U, K Ranguelova, R Kappl, Jürgen Hüttermann, Susanne Fetzner (2004) Dioxygenases without Requirement for Cofactors and Their Chemical Model Reaction: compulsory order ternary complex mechanism of 1H-3-hydroxy-4-oxoquinaldine 2,4-dioxygenase involving general base catalysis by histidine 251 and single-electron oxidation of the substrate dianion. Biochem 43(45): 14485-14499.

53. Orville AM, Lountos GT, Finnegan S, Giovanni Gadda, Rajeev Prabhakar (2009) Crystallographic, Spectroscopic, and Computational Analysis of a Flavin C4a-Oxygen Adduct in Choline Oxidase. Biochem 48(4): 720-728.

54. Khersonsky 0, Tawfik DS (2010) Enzyme promiscuity: a mechanistic and evolutionary perspective. Annu Rev Biochem 79: 471-505.

55. Bugg TDH (2014) How to Break the Rules of Dioxygen Activation. Chem Biol 21(2): 168-169.

56. Parvez S, Long MJC, Poganik JR, Aye Y (2018) Redox Signaling by Reactive Electrophiles and Oxidants. Chem Rev 118(18): 8798-8888.

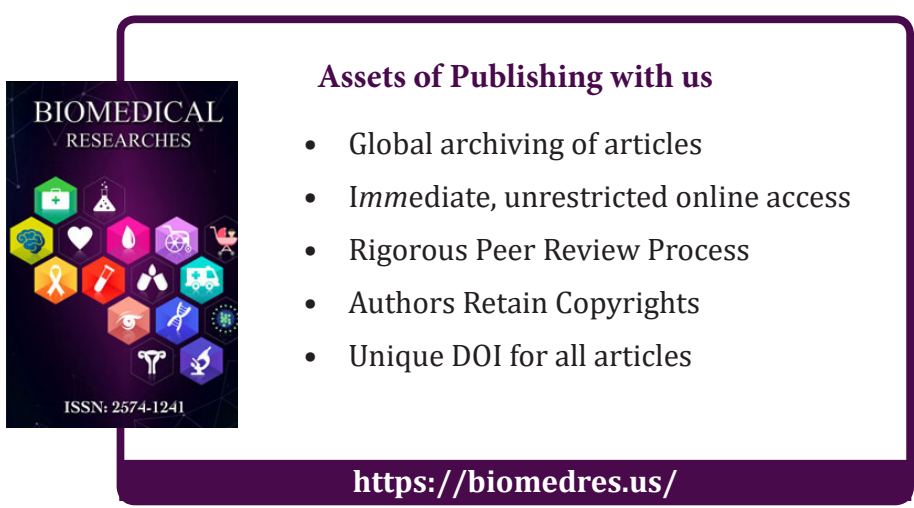

